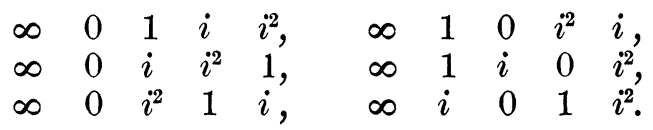

For $p^{n}=5$, the only group solution is one equivalent to that by C. H. Judson in the American Mathematical Monthly, 1900, page 72. For $p^{n}=7$ there are exactly four group solutions; for $p^{n}=9$, there are four. The second group $H_{72}$ actually furnishes solutions.

15. The second paper by Professor Dickson relates to the question of redundancies in Professor Moore's set of relations defining $G$ for the $G F\left[p^{n}\right]$. It is an addition to a paper with the same title in the Proceedings of the London Mathematical Society, volume 35 (1902), pages 292-305, which treated the cases for which $p^{n} \leqq 48$. In the present paper, the theorem of the paper cited is proved, by fortunate devices, to hold for the two new cases $p^{n}=3^{5}$ and $p^{n}=5^{3}$.

Evanston, IIL., April 15, 1904.
Thomas F. Holgate, Secretary of the Section.

\title{
THE HEINE-BOREL THEOREM.
}

BY DR. OSWALD VEBLEN.

This note has for its main object a proof that the Heine-Borel theorem is equivalent, as a continuity axiom, to the Dedekind cut proposition.* In $\$ 3$ the equivalence of the closure of a set with what may be called the $\mathrm{H}-\mathrm{B}$ property is shown to apply not only to the continuum but to any set of numbers. $f$ This result applies in manifolds of any number of dimensions as well as to linear sets.

1. I. If every number of an interval $A B$ belongs to at least one segment $\sigma$ of a set of segments $[\sigma]$, then there exists a finite sub-

* The equivalence in question suggested itself to Mr. N. J. Lennes and myself while we were working over some elementary propositions in real function theory.

† This extends a theorem of Dr. W. H. Young to the effect that every closed linear set has the H-B property. Cf. W. H. Young, "Overlapping Intervals." Proceedings of the London Mathematical Society, p. 384, vol. 35 (1903). 
set of $[\sigma], \sigma_{1}, \sigma_{2}, \sigma_{3}, \cdots, \sigma_{n}$, such that every number of $A B$ belongs to at least one of the segments $\sigma_{1}, \sigma_{2}, \cdots \sigma_{n}$.

By a segment $A B=\sigma(A<B)$ is meant the set of real numbers $[X]$ such that $A<X<B$. By an interval $A B$ is meant the set of real numbers $[X]$ such that $A \leqq X \leqq B$. Proposition I is essentially involved in Heine's * proof of the theorem of uniform continuity, but has first been stated in explicit form by Borel, $\uparrow$ who was careful to specify that the set of segments of which he spoke should be at most numerably infinite. The theorem, however, is valid whatever be the set $[\sigma]$, and indeed to the restricted form given it by Borel the remarks below do not wholly apply.

In its generalized form, the Heine-Borel theorem can be used to replace the tedious " caging" process which appears so frequently in rigorous analysis. As an example, let us take Weierstrass's theorem that any infinite set of numbers $[X]$ lying on a bounded interval $A B$ has at least one limit number. If the theorem should fail, then about each number of the interval $A B$ there would be a segment $\sigma$ containing not more than one number of the set $[X]$. By the Heine-Borel theorem, there would exist a finite subset $\sigma_{1}, \sigma_{2}, \ldots, \sigma_{n}$ of these segments $\sigma$ such that every number of $A B$ would lie on at least one such segment. But as each of $\sigma_{1}, \sigma_{2}, \cdots, \sigma_{n}$ contained only one number of $[X],[X]$ could contain only a finite number of numbers, contrary to hypothesis.

2. Besides being of great convenience in the routine of limiting processes, the generalized form of the Heine-Borel theorem is equally fundamental with the Dedekind cut proposition as an axiom of continuity for the real number system. The Dedekind statement is as follows:

II. If all the numbers of an interval $A B$ fall into two classes $[M]$ and $[N]$, such that every $M$ is greater than every $N$, then there exists a number $O$ such that $M \geqq O \geqq N$ for every $M$ and every $N$.

The assertion that I and II are equally fundamental may be more exactly stated as follows :

III. Admitting the ordinal relations of the real number system, I is a consequence of II and II is a consequence of $\mathrm{I}$.

* Heine, "Die Elemente der Functionenlehre," Crelle, vol. 74 (1872), p. 188.

†Borel, "Sur quelques points de la théorie des fonctions," Annales de l'École Normale Supérieure, 3d series, vol. 12 (1895), p. 51. 
The proof that I is a consequence of II is well-known* and is given in a generalized form below. To prove II from I, observe that if there is no number $O$ of the kind required, [M] has no greatest lower bound and $[N]$ no least upper bound. Therefore, about each number $M$ there is a segment not including any number $N$, and about each number $N$ a segment not including any number $M$. Every number of $A B$ being either an $M$ or an $N$, this set of segments satisfies the hypothesis of $\mathrm{I}$. Hence there is a finite set of segments $\sigma_{1}, \sigma_{2}, \ldots, \sigma_{n}$ including every number of $A B$ and each including only numbers of $[M]$ or only numbers of $[N]$. Let $A$ be the smaller of $A$ and $B$. Of the finite number of segments which include $A$, no one includes or is bounded by a number of $[M]$. Let the largest bound of the segments including $A$ be $A^{\prime}$. Proceed with $A^{\prime}$ (which is a number of $[N]$ ) as with $A$, obtaining $A^{\prime \prime}$ of $[N]$ and so on. Since there are a finite number of segments $\sigma_{1}, \cdots, \sigma_{n}$, the process leads by a finite number of steps to the conclusion that none of the segments include numbers of $[M]$, contrary to bypothesis.

3. The Heine-Borel theorem has a generalization of some importance in the theory of sets. Let us say that a set of real numbers $[X]$ has the H-B property if, from every set of segments $[\sigma]$ such that every number $X$ belongs to at least one $\sigma$, there can be selected a finite subset $\sigma_{1}, \sigma_{2}, \ldots, \sigma_{n}$ such that every $X$ belongs to at least one $\sigma_{k}(k=1,2, \ldots, n)$. By a closed set, let us mean one that includes all its limit numbers.

IV. $A$ necessary and sufficient condition that a bounded set $[X]$ of numbers shall have the $H-B$ property is that $[X]$ is closed.

It is evident that an infinite set not including its limit numbers has not the $\mathrm{H}-\mathrm{B}$ property. For if $[X]$ lies on $A B$ $(A<B)$ and has a limit number $C$, not an $X$, let $[\underline{C}]$ be the set of all numbers smaller than $C$, and let $[\bar{C}]$ be the set of all numbers larger than $C$. The set of segments, $A \underline{C}, B \bar{C}$ has no finite subset including every number $X$.

In proving that a closed set has the $\mathrm{H}-\mathrm{B}$ property, we assume without loss of generality that $A<B$ and that $A$ and $B$ belong to $[X]$. Either, one of the segments $\sigma=A_{0} A_{1}$ includes $A B$, in which case $n=1$, or the ends [ $\left.A^{\prime}\right]$ of the seg-

* Cf. Schoenflies, "Bericht über die Mengenlehre," Jahresbericht der Deutschen Mathematiker-Vereinigung, vol. 8 (1899), p. 51. 
ments including $A$ are all less than $B$. It is an immediate and well-known consequence of II that every bounded set of numbers has a least upper bound. Let $A_{1}$ be the least upper bound of $\left[A^{\prime}\right]$. Either $A_{1}$ belongs to the set $[X]$ or, by the closure of $[X]$, one of the segments including $A$ includes all numbers $X$ between $A$ and $A_{1}$. If $A_{1}$ is an $X$, we denote it by $X_{1}$, if not, let $X_{1}$ be the smallest number $X$ (existent because $[X]$ is closed) which is greater than $A_{1}$. Proceed with $X_{1}$ as with $A_{1}$, obtaining $A_{2}$ and, if necessary, $X_{2}$. By a finite number of steps, we obtain $A_{k} \geqq B$; for, if not, the bounded set $X_{1}, X_{2}, \ldots$ would have a least upper bound $X_{\omega}$ which, because of the closure of $[X]$, would be a limit number of $[X]$. Hence there would be a segment $\sigma$ including $X_{\omega}$; this segment must include at least one $X_{m}$ and, therefore $A_{m+1}$, contrary to the hypothesis that $A_{m+1}$ is the least upper bound of the ends of the segments including $X_{m}$.

To obtain the set $\sigma_{1}, \ldots, \sigma_{n}$, let $\sigma_{1}, \ldots, \sigma_{k}$ be a set of segments of $[\sigma]$ including $X_{1}, X_{2}, \cdots, X_{k-1}, B$; let $\sigma_{k+1}$ be the segment of $[\sigma]$ including $A$ and all numbers of $[X]$ between $A$ and $X_{1}$ in case $A_{1} \neq X_{1}$ and a segment of $[\sigma]$ including $A$ and the lower end of $\sigma_{1}$ in case $A_{1}=X_{1}$; similarly, let $\sigma_{k+h}(h=2, \cdots, k)$ include $X_{h-1}$ and all numbers between $X_{h-1}$ and $X_{h}$ if $X_{h} \neq A_{h}$ or include $X_{h-1}$ and the lower end of $\sigma_{h}$ if $A_{h}=X_{h} . \quad n=2 k$ (unless $n=1$ as above).

4. The theorems proved above depend upon the linear arrangement and continuity of the real number system and not at all upon the operations of addition and multiplication. They therefore apply equally well in the geometry of a straight line, even without metric hypotheses, and in fact to any interval of a continuous curve without multiple points.

The validity of the Heine-Borel theorem for the plane (or for space of any number of dimensions) suggests that it can be used as a continuity axiom, not only for real numbers, but also for complex number systems.

The University of Chicago, March 26, 1904. 